\title{
Realization of Distributed Communication Simulation Framework
}

\author{
Lin Jianning, Yu Jie \\ Science and Technology on Information Systems Engineering Laboratory, Nanjing 210007, China, \\ China \\ e-mail: oliver_ljn@126.com
}

Keywords: communication framework; distributed simulation; communication efficiency

\begin{abstract}
According to the requirement of communication link simulation in the process of simulation experiment, this paper designs a framework of distributed communication simulation. The framework can dynamically load communication efficiency calculation model which exists in the form of a DLL for different simulation demands. Then this paper introduces the deployment, initialization and running of the distributed framework. Finally, this paper proposes a communication efficiency calculation model based on the link state change with less CPU resource and higher real time.
\end{abstract}

\section{Introduction}

Today, distributed simulation systems are mostly created following high level architecture (HLA) ${ }^{[1]}$ which is kind of simulation federation system customized and developed to finish specific simulation tasks, and HLA can keep the independence of federation members as well as realize effective interaction of information. In the distributed simulation system, all federation members are deployed in different simulation nodes and communication links between different simulation nodes will be simulated in order to enhance the authenticity of information simulation in the simulation system and make it closer to the actual system.

At present, studies on communication framework are mostly based on the structure of actual network communication link. In 2007, instant message system ${ }^{[2]}$ based on $\mathrm{B} / \mathrm{S}$ and $\mathrm{C} / \mathrm{S}$ was proposed, and Ajax and other technologies were chosen as the client-side implementing mechanism to mainly serve the information interaction between actual systems; in 2010, system communication framework $^{[3]}$ based on Agent was proposed, and XML and SOAP technical architectures are used to focus on solving the transfer of different network transport protocols in the process of system information interaction; in 2013, network communication system ${ }^{[4]}$ based on middle ware was proposed to reduce the design complexity of network communication and realize real-time and reliable network communication.

In the field of simulation, communication efficiency model study is generally used to realize communication framework simulation ${ }^{[5]}$. The communication efficiency model of the whole communication network is rather complex and difficult, and the effective solution is to design the communication efficiency model of all links between simulation nodes, and simulate the communication efficiency of the whole network through the calculation and synchronization of link communication efficiency parameters ${ }^{[6][7]}$.

\section{Distributed communication simulation framework}

\section{Framework architecture}

Distributed communication simulation framework adopts $\mathrm{C} / \mathrm{S}$ architecture, and includes communication simulation framework calculation server and communication simulation framework client, as shown in Fig.1. Communication simulation framework calculation server includes deployment module of communication model, initialization module of communication model, calculation request processing module of communication model and synchronization module of communication simulation framework client, and these modules are generally deployed in the 
simulation nodes to meet the demand of communication simulation efficiency calculation. Communication simulation framework client is deployed in the simulation nodes, and includes synchronization module of calculation server, communication simulation effect feedback module and mode state report module.

Communication simulation efficiency calculation model shall be deployed in the communication simulation framework in advance for the automatic loading and configuration of the communication framework during the running process. Different communication efficiency calculation models can be deployed in the communication simulation framework, and each one is a DLL file which is the executive program for the model to process logics. Each communication efficiency calculation model must provide the model description file in XML format. The description file includes initialization parameter list of the model, stored information of the initialization parameter, model ID, corresponding simulation node ID of the model, and model interface parameter, etc.

\section{Framework work flow}

1) Deployment process

After finishing the development of communication simulation efficiency calculation model, it is deployed in the communication framework via the deployment module of the communication model to be used in the simulation experiment. The deployment module of the communication model has deployment, anti-deployment and re-deployment functions; module deployment means that the developed communication efficiency calculation model is loaded in the communication simulation framework, so as to be called; model anti-deployment means that the deployed simulation communication efficiency calculation model is removed from the communication simulation framework; module re-deployment means that the deployed older version of simulation communication efficiency calculation model is updated. As shown in diagram 2, the deployment module of the communication model is a series of operation processes for the model file, and includes local file monitoring function, framework model deployment file, model deployment, antideployment and re-deployment functions. Local file monitoring is used for downloading remote module files and managing local module files, and the deployment module of the communication model will automatically deploy, anti-deploy and re-deploy model files according to framework model configuration files.

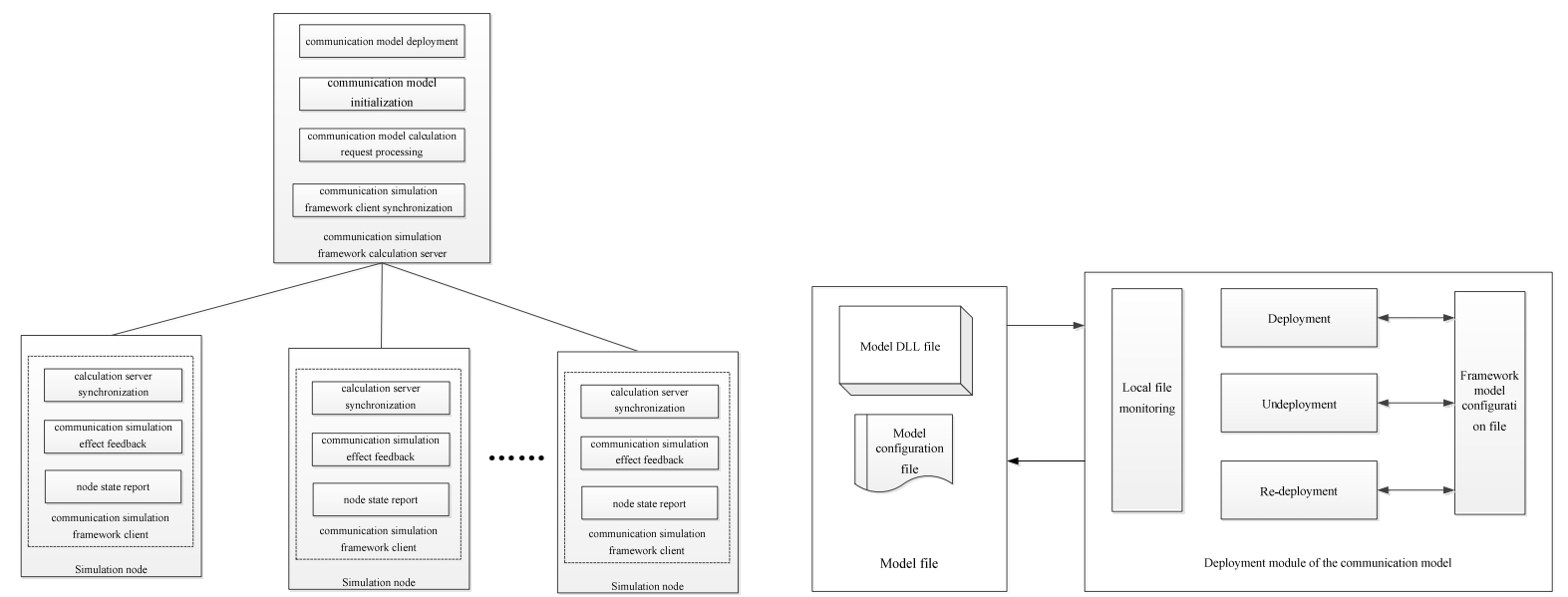

Fig 1 Composition diagram of communication simulation framework

Fig 2 Deployment Process Diagram

In the process of the simulation experiment, different communication efficiency parameter calculation models will be used according to different network simulation requirements Therefore, in the initialization, the communication simulation framework calculation server can automatically deploy the communication simulation efficiency calculation model used in the simulation experiment according to the requirement of the simulation experiment system.

After the successful start of the communication simulation framework server, read and parse the configuration file of the communication simulation module; store the communication simulation efficiency calculation model list requiring deployment at present and the deployment parameter information of each communication model; deploy the communication simulation efficiency 
calculation model according to the deployment parameters, and see the specific deployment process shown in Fig. 3.

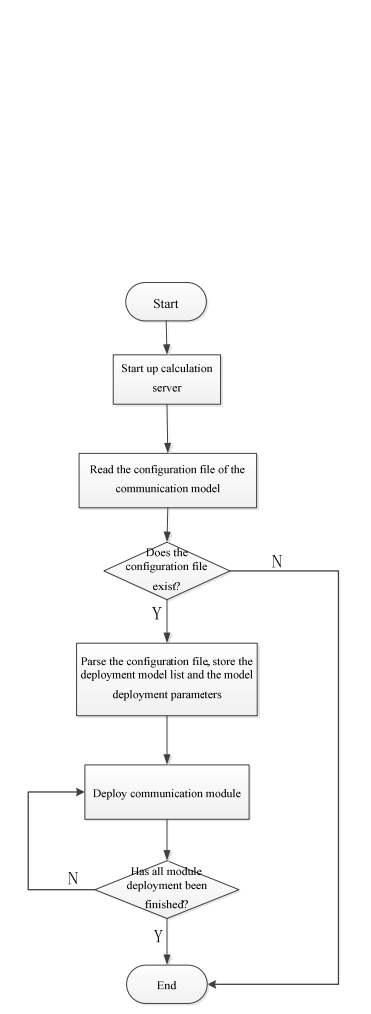

Fig 3 Model loading process diagram

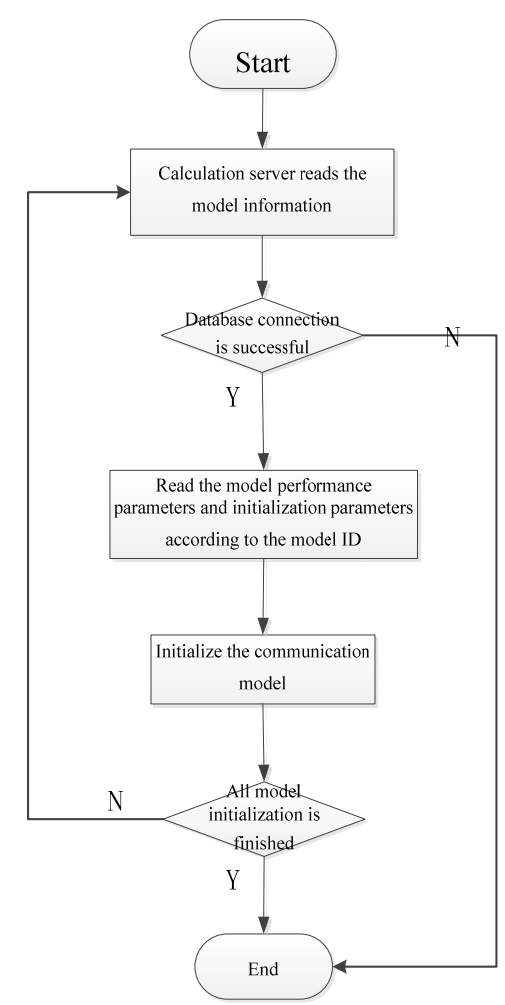

Fig 4 Model initialization process of computation server

2) Initialization process

After the successful start of the communication simulation framework server, read and parse the configuration file of the communication simulation module; store the communication simulation efficiency calculation model list requiring deployment at present and the deployment parameter information of each communication model; deploy the communication simulation efficiency calculation model according to the deployment parameters, and see the specific deployment process shown in Fig. 4.

3) Running process

After the initialization of the communication efficiency calculation model, the calculation server of the communication simulation framework will traverse the communication links neighboring the simulation nodes in turn, match the initialized communication efficiency calculation model according to the simulation nodes on both ends of the link, and send the calculation request to calculate the communication efficiency parameters of each communication link to be simulated; after calculation, the calculation server of the communication simulation framework will synchronize the calculation results to the communication simulation framework client on the simulation nodes on both ends of the link. After receiving the communication efficiency parameters, the client will superimpose the communication efficiency parameters on the message in each link through such node, and when the communication efficiency parameters of the communication link change, the calculation server will calculate and synchronize them according to the new link performance parameters, as shown in diagram 5.

During the simulation experiment, the performance parameters of the communication link will change, and the communication efficiency parameters of one link will follow when the performance parameters of some communication link change. Therefore, it is required to calculate the communication efficiency parameters again according to the new performance parameters of the link. To change the performance parameters of the communication link, you can manually enter and modify the parameters at the human-computer interaction interface provided by the said state update sub-module in the calculation server, or you can report the communication link status after 
simulation node change to the calculation server through the said node state reporting module in the communication framework client, as shown in Fig. 6.

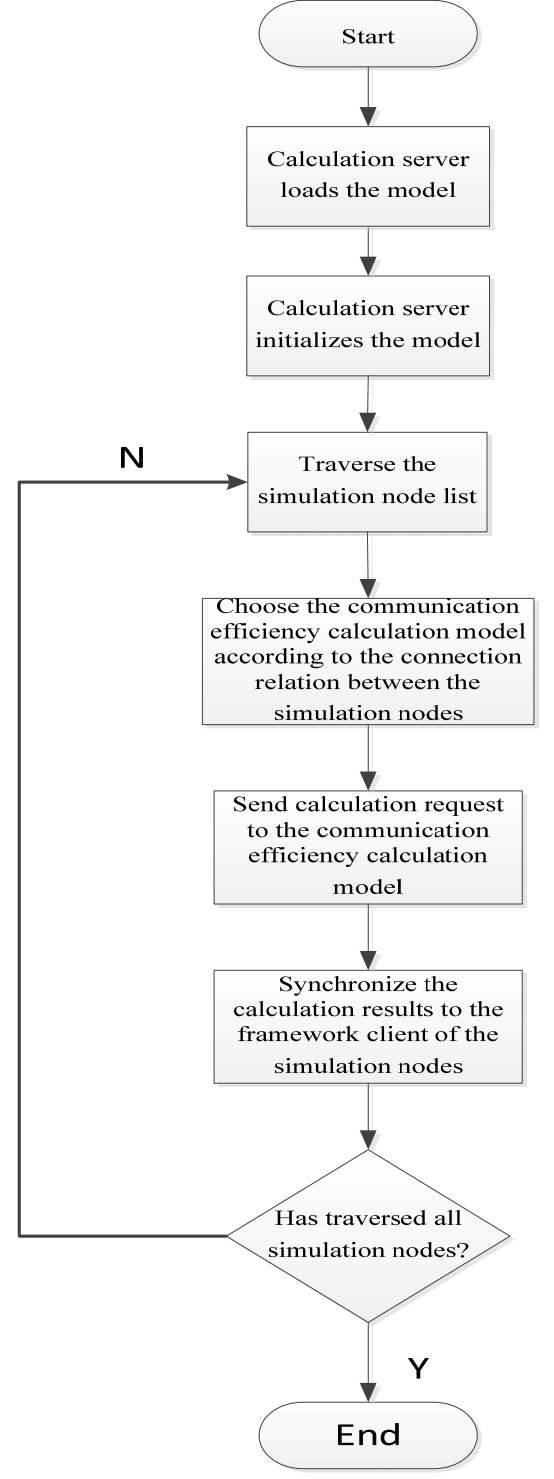

Fig 5 Communication efficiency calculation synchronization process

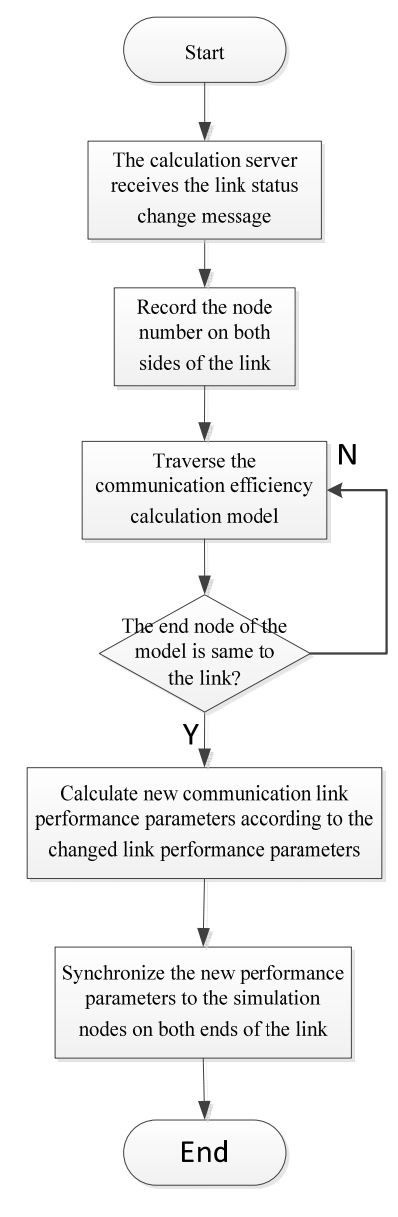

Fig 6 Calculation parameters updating process

a) The calculation server receives the message on link status change, and stores this message in the status change message queue;

b) The said client synchronization module of the communication simulation framework records the simulation node number on both ends of the communication link;

c) The said client synchronization module of the communication simulation framework traverses the stored communication efficiency calculation model, and match the node numbers on both ends of the communication link recorded in step 2 with the simulation node link of such communication efficiency calculation model;

d) If the communication link end node the model corresponds to is same to the changes simulation node, calculate the communication performance parameters after link change according to the changed link performance parameters; otherwise, continue traversal and returns to step 3;

e) The said client synchronization module of the communication simulation framework synchronizes the changed communication efficiency parameters to the communication framework client on the simulation node on both sides of the link

f) End the synchronization process. 


\section{Experiment results and analysis}

According to the above-mentioned distributed communication simulation framework, this paper realizes a distributed simulation system which is based on HLA platform and includes 12 federation members, and the experiment environment is Intel Core $23.0 \mathrm{GHz}$ CPU, 2GB RAM.

The distributed communication simulation framework realized in this paper includes 1 scenario generation node, 8 intelligence detection nodes, 2 intelligence treatment nodes and 1 battlefield situation node. In the process of simulation, the battle occurrence node sends battlefield information to the intelligence detection nodes which report the detected intelligence information to intelligence treatment nodes which send the treated intelligence information to battlefield situation node for situation display. The communication efficiency calculation model deployment of the links between simulation nodes is shown in Fig.7.

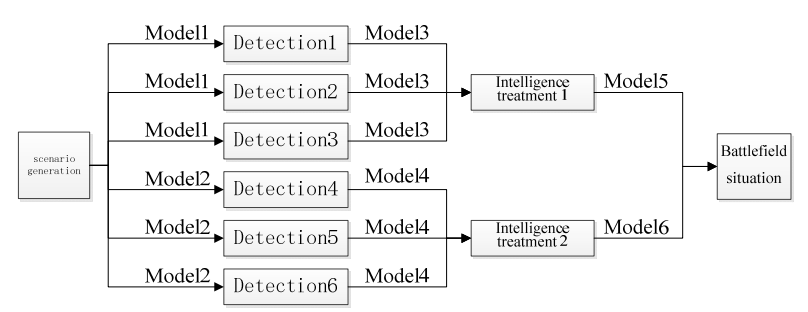

Fig 7 Communication efficiency calculation model deployment of the distributed simulation system

The simulation experiment proves that the distributed communication simulation framework can automatically deploy and initialize the communication efficiency calculation model. During the simulation process, the performance parameters of all communication links can be intervened manually. The communication simulation framework can calculate and update the performance parameters of the communication link in time according to the status change of communication links, and the intelligence changing with the network performance change can be observed on the battlefield situation. In terms of calculation resources, CPU occupancy rates of communication simulation framework calculation server and all clients of the communication simulation framework are below $10 \%$.

\section{Conclusion}

This paper designs a distributed communication simulation $g$ framework based on modules, and can automatically select the communication efficiency calculation model according to experiment requirement and link status, and support communication link simulation of various types. At the same time, in the communication efficiency calculation framework, synchronization at one time just need to consider the communication efficiency simulation matching such simulation node, and avoids concurrent calculation of many communication efficiency simulation models, which can reduce computing resources cost, improve the real time of communication link efficiency calculation, and meets the requirement of performance simulation in the simulation system communication.

\section{References}

[1] The Institute of Electrical and Electronics Engineers: IEEE Standard 1516 for Modeling and Simulation (M\&S) High Level Architecture (HLA) ---Framework and Rules, http://www.ieee.org, 2000

[2] Escoffier C, Hall R S, Lalanda P.IPOJO: An extensible server oriented component framework[C] // 2007 IEEE International Conference on Servers Computing, Salt lake City: IEEE Computer society, 2007:474-481

[3] Li Xiaoyu, Yu Qian. A multi-Agent system communication framework [J], Journal of Chongqing Institute of Technology, Vol24 (5), 2010, 100-104

[4] Pullen J.M., Brunton R., Brutzman D., Drake D., Hieb M., Morse K.L., Tolk A. Using Web 
Services to Integrate Heterogeneous Simulations in a Grid Environment [A]. In Proceedings of the International Conference on Computational Science, Krakow, Poland, 2004.

[5] Lv Zixu, Zhang Jiong. Network communication based on TAO middle ware in the distributed simulation system [J], Journal of Jinan University, Vol27 (1), 2013, 47-51

[6] Zajac K, Bubak M, Malawski M, Sloot P.M. A. Towards a grid management System for HLA-based interactive simulations [A]. In Proceedings of Seventh IEEE International Symposiums on Distributed Simulation and Real-Time Applications[C].23-25 Oct, 2003.

[7] Li B H, et al. New Distributed Collaborative Simulation System-Simulation Grid [J]. Journal of System Simulation, Vol 20,No 20,Oct,2008.

[8] Li Bo-hu, Chai Xu-dong. Networked Modeling \& Simulation Platform Based on Concept of Cloud Computing ----Cloud Simulation Platform Journal of System Simulation, Vol.21,No.17, Sep,2009 